\title{
Evaluation of the effect of standard neuronavigation and augmented reality on the integrity of the perifocal structures during a neurosurgical approach
}

\author{
Alioucha Davidovic, ${ }^{2}$ Lara Chavaz, ${ }^{2}$ Torstein R. Meling, MD, DPhil, ${ }^{1,2}$ Karl Schaller, MD, ${ }^{1,2}$ \\ Philippe Bijlenga, MD, PhD, ${ }^{1,2}$ and Julien Haemmerli, MD' ${ }^{1}$ \\ 1Division of Neurosurgery, Department of Clinical Neurosciences, Geneva University Hospitals; and 'Faculty of Medicine, \\ University of Geneva, Geneva, Switzerland
}

\begin{abstract}
OBJECTIVE Intracranial minimally invasive procedures imply working in a restricted surgical corridor surrounded by critical structures, such as vessels and cranial nerves. Any damage to them may affect patient outcome. Neuronavigation systems may reduce the risk of such complications. In this study, the authors sought to compare standard neuronavigation (NV) and augmented reality (AR)-guided navigation with respect to the integrity of the perifocal structures during a neurosurgical approach using a novel model imitating intracranial vessels.
\end{abstract}

METHODS A custom-made box, containing crisscrossing hard metal wires, a hidden nail at its bottom, and a wooden top, was scanned, fused, and referenced for the purpose of the study. The metal wires and an aneurysm clip applier were connected to a controller, which counted the number of contacts between them. Twenty-three naive participants were asked to 1) use NV to define an optimal entry point on the top, perform the smallest craniotomy possible on the wooden top, and to use a surgical microscope when placing a clip on the nail without touching the metal wires; and 2) use AR to preoperatively define an ideal trajectory, navigate the surgical microscope, and then perform the same task. The primary outcome was the number of contacts made between the metal wires and the clip applier. Secondary outcomes were craniotomy size, and trust in NV and AR to help avoid touching the metal wires, as assessed by a 9-level Likert scale.

RESULTS The median number of contacts tended to be lower with the use of AR than with NV (AR, median 1 [Q1: 1, Q3: 2]; NV, median 3 [Q1: 1, Q3: 6]; $p=0.074$ ). The size of the target-oriented craniotomy was significantly lower with the use of AR compared with NV (AR, median $4.91 \mathrm{~cm}^{2}$ [Q1: $4.71 \mathrm{~cm}^{2}, \mathrm{Q} 3: 7.55 \mathrm{~cm}^{2}$ ]; and NV, median $9.62 \mathrm{~cm}^{2}$ [Q1: 7.07 $\mathrm{cm}^{2} ;$ Q3: $\left.13.85 \mathrm{~cm}^{2}\right]$ ). Participants had more trust in AR than in NV (the differences posttest minus pretest were mean 0.9 [SD 1.2] and mean -0.3 [SD 0.2], respectively; $p<0.05$ ).

CONCLUSIONS The results of this study show a trend favoring the use of AR over NV with respect to reducing contact between a clip applier and the perifocal structures during a simulated clipping of an intracranial aneurysm. Target-guided craniotomies were smaller with the use of AR. AR may be used not only to localize surgical targets but also to prevent complications associated with damage to structures encountered during the surgical approach.

https://thejns.org/doi/abs/10.3171/2021.5.FOCUS21202

KEYWORDS augmented reality; neuronavigation; safety task

$\mathrm{D}$ URING minimally invasive intracranial approaches, the surgeon has to deal with critical structures, such as the vessels and the cranial nerves along the restricted and narrow surgical corridors. Any neurovascular injury during the surgical approach may dramatically affect patient outcome. The development of preoperative image-based neuronavigation systems have allowed a reduction in postoperative patient morbidity due to the abil- ity to adapt the surgical strategy intraoperatively to respect healthy tissues. ${ }^{1-5}$

Although standard neuronavigation (NV) offers a permanent localization of structures of interest intraoperatively, it has several disadvantages; the neurosurgeon must look at dedicated NV screens, transpose 2D images onto 3D real images through the surgical microscope, and turn his or her focus away from the surgical field. ${ }^{2,6}$ The use of 


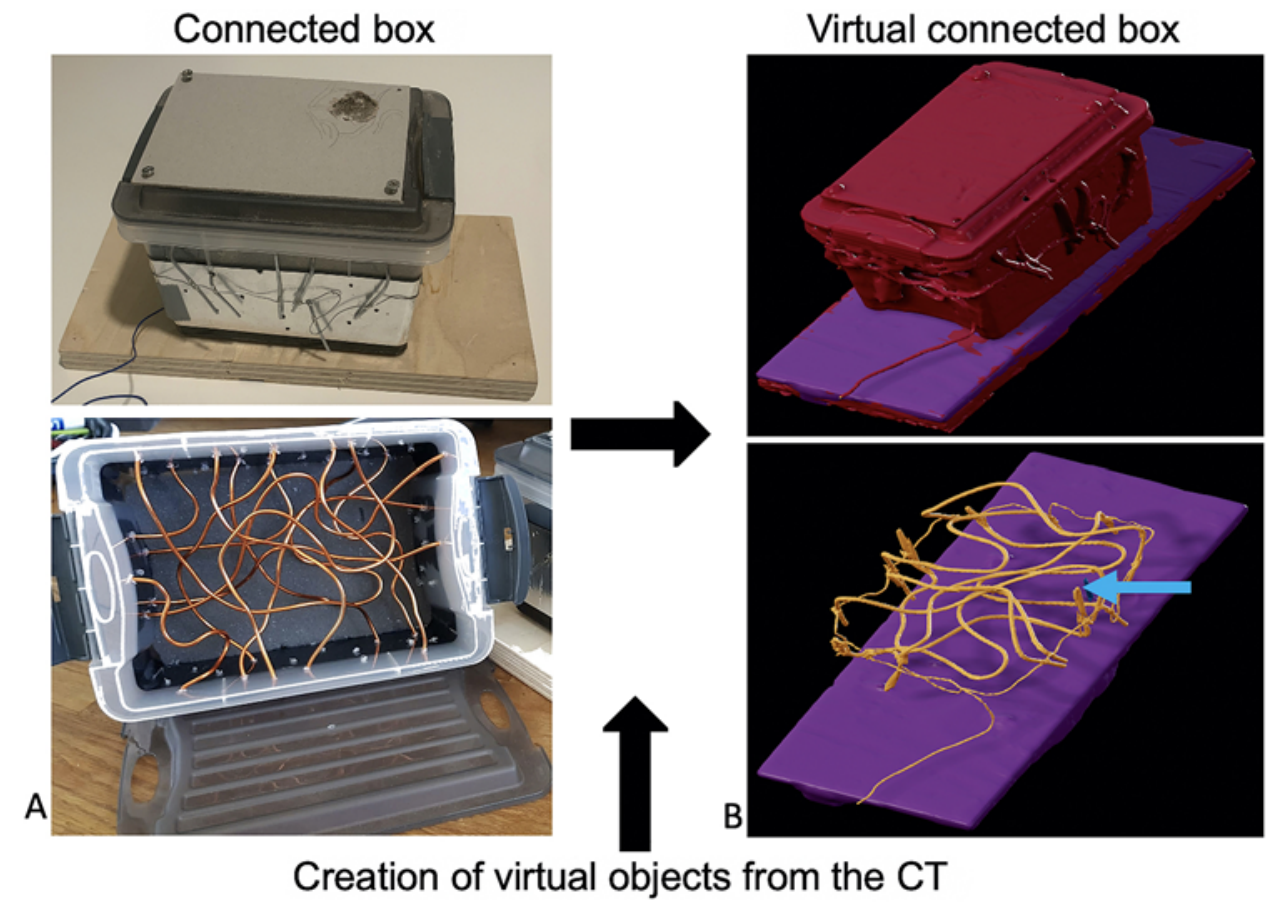

FIG. 1. A: A plastic box was made for the study. Hard metal wires were inserted into the box at random, each with a unique trajectory. The bottom of the box was filled with foam to hide the target, a 4-cm nail. B: The box was scanned in a CT machine, and virtual objects were defined from the CT images for the AR task.

the augmented reality (AR), or the projection of preregistered virtual objects through the surgical microscope, alleviates the limitations of $\mathrm{NV} ;^{2}$ the surgeon keeps his or her focus on the surgical field and has a direct 3D rendering of the virtual structures. AR is gaining interest not only in intracranial tumor surgeries ${ }^{6-8}$ but also during cerebrovascular $^{9-12}$ and spinal surgeries. ${ }^{13-15}$

In a recently published experimental study, we analyzed the precision of NV and AR-assisted navigation by measuring the distance between the virtual projected image and the real target. Therein, we showed the superiority of AR over NV regarding the precision-to-target, due to the permanent possibility to recalibrate the AR according to the signature structures. ${ }^{2}$ Although precision is an integral component of surgical safety, our experiment did not analyze the integrity of the perifocal structures with the use of NV and AR.

In this study, we used a novel model mimicking a microsurgical corridor to evaluate the integrity of the perifocal structures during a typical aneurysm clipping procedure, which we dubbed "the safety tasks," using NV and AR. We hypothesized that the use of AR could enhance the procedural safety by reducing the number of contacts with the perifocal structures, and by reducing the size of the craniotomy, compared with NV.

\section{Methods}

\section{Connected Wire Box and Clip Applier}

The NV and AR safety tasks were similar to those in the board game Operation (Hasbro) in that they were designed to give a sound alert if there was any contact between the surgical instrument and a metal wire that represented an intracranial vessel.

A $140 \times 95 \times 90-\mathrm{mm}$ plastic box was made for the experiment (Fig. 1A). Hard metal conducting wires were inserted randomly inside the box to mimic vessels. Each wire had a tortuous and unique trajectory. At a bottom corner of the box, a 4-cm metal nail was placed. The bottom of the box was covered by foam so that only the tip of the nail was visible. The top of the box consisted of a rectangular wooden plate with a 4-screw fixation system, so that it was replaceable. A standard aneurysm clip applier (Peter LAZIC GmbH) with a standard 7-mm straight aneurysm clip was used. An electric cable was fixed to its proximal end. The Arduino UNO microcontroller (Arduino) connected the network of metal wires inside the box and an electric cable from the clip applier, such that any contact between the clip applier and a wire was recorded and announced to the participant with a sound alert.

\section{Microcontroller and Software}

The Arduino UNO is an open-source hardware microcontroller using the C-programming language. It was used as an interface to connect the metal wires inside the box and the clip applier to a computer. The number of contacts between the metal wires and the clip applier was recorded and counted using Arduino IDE version 1.8.10 (Arduino) software. To prevent unwanted multiple counts, a 100msec refractory time was set between two contacts. The code in $\mathrm{C}$ is available on request. 


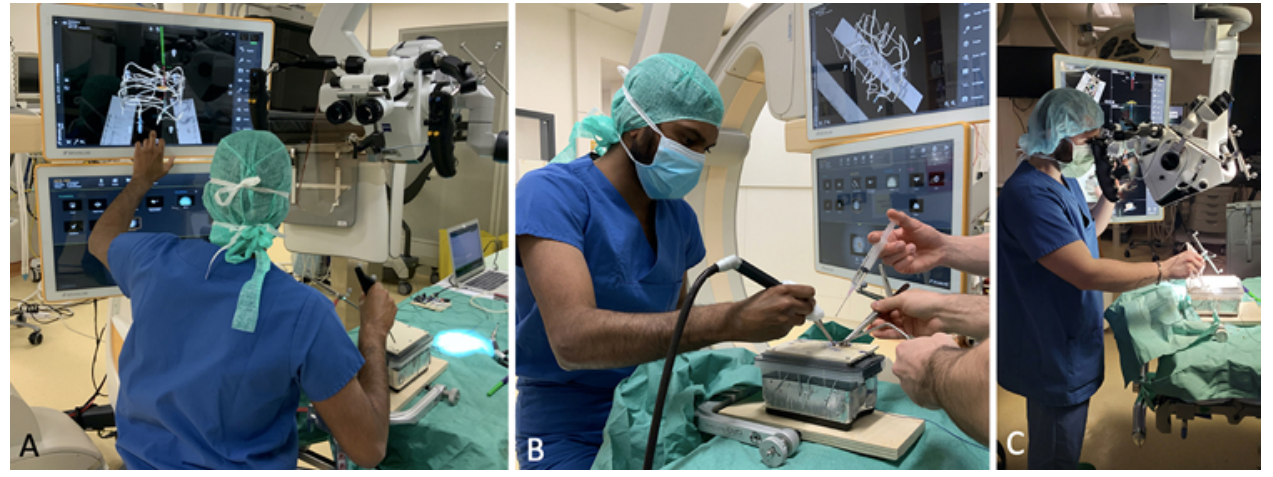

FIG. 2. The safety task using NV. A: Participants were asked to define an entry point according to the target and the metal wires. B: The wooden top of the box was drilled according to NV. C: Under microscope, participants were asked to clip the target (nail) without touching the metal wires with the help of the navigation pointer.

\section{NV and AR Registration}

The wire box was scanned, and from the CT images, Elements (Brainlab) software was used to create virtual objects such as the box borders, metal wires, and the nail (target) (Fig. 1B). The wire box was registered preoperatively with a Brainlab NV system using 6 predefined points.

For the purpose of AR, the surgical microscope (Zeiss Kinevo 900, Carl Zeiss Meditec) was navigated and referenced after focusing on the center of the referencing array. Projected virtual objects were then adjusted to the real structures using AR software (Elements, Application Microscope, Brainlab).

\section{Participants}

Twenty-three participants took part in the study. They were all medical students from the Geneva Faculty of Medicine. All participants performed the safety tasks using both $\mathrm{NV}$ and $\mathrm{AR}$.

\section{Setup and Tasks}

The tasks were performed in a standard surgical condition as previously described, ${ }^{2}$ with the aim to mimic a minimally invasive surgical approach through a limited craniotomy size. The wire box was held in a Mayfield head clamp with the reference array affixed to it. Participants received a brief introduction on the use of NV and $\mathrm{AR}$, with an opportunity to try the systems for 10 to 15 minutes before performing the tasks.

The first task was performed using NV and a navigated pointer (Fig. 2). Referencing of the wire box was performed using 6 predefined landmarks on its surface by the senior author. Participants were asked to 1) localize the target with NV; 2) choose an entry point on the wooden top of the wire box; 3) perform a circular craniotomy as small as possible using a high-speed drill; and 4) use the microscope and the navigation pointer as aids when navigating the clip applier through the maze of metal wires and place an aneurysm clip on the hidden body of the nail without touching the metal wires.

The second task was performed using AR and a navigated microscope (Fig. 3). The coregistration and recal- ibration of the microscope was performed by the senior author. Using the Elements neuronavigation software, the participants were first asked to identify an ideal trajectory between an entry point on the wooden top of the wire box to the target at a reasonable distance to the metal wires. Then the participants were asked to 1) place the microscope according to the planned trajectory using AR, 2) perform a circular craniotomy as small as possible using a high-speed drill, and 3) use the AR when clipping the hidden body of the nail without touching the metal wires.

For both safety tasks, the number of attempts was recorded (one attempt was defined by one entry with the clip applier inside the box). The surface of the craniotomy was calculated and expressed in square centimeters. The Arduino UNO microcontroller counted the number of contacts between the clip applier and the metal wires. Finally, successful clipping of the hidden body of the nail was noted.

Before and after realizing each task, all participants were asked "On a scale from 0 to 9 , how much do you trust the technology to reach a small target?" (for both AR and NV), with 0 being no trust and 9 being complete trust (9-level Likert scale). Assessments before and after the experiment were compared (pretest and posttest trust).

\section{Statistical Analysis}

Statistical analyses were performed with the use of RStudio and R version 3.6.1 (https://www.r-project.org) software. All continuous nonparametric variables were compared using a two-way Wilcoxon signed-rank test. Results were expressed in terms of median, first quartile $(\mathrm{Q} 1)$, and third quartile $(\mathrm{Q} 3)$. A p value $<0.05$ was considered significant. According to the pilot study, we hypothesized that AR would reduce the number of contacts by twofold. The power calculation revealed that 13 participants per group were needed to reach an $80 \%$ chance to detect a twofold reduction in the number of contacts, considering an alpha error of 5\%. Similarly, we hypothesized that AR would decrease the size of the craniotomy by $20 \%$. The power calculation determined that a sample size of 17 participants per group was necessary to reach an $80 \%$ chance to detect a $20 \%$ reduction in the craniotomy size, considering an alpha error of $5 \%$. 

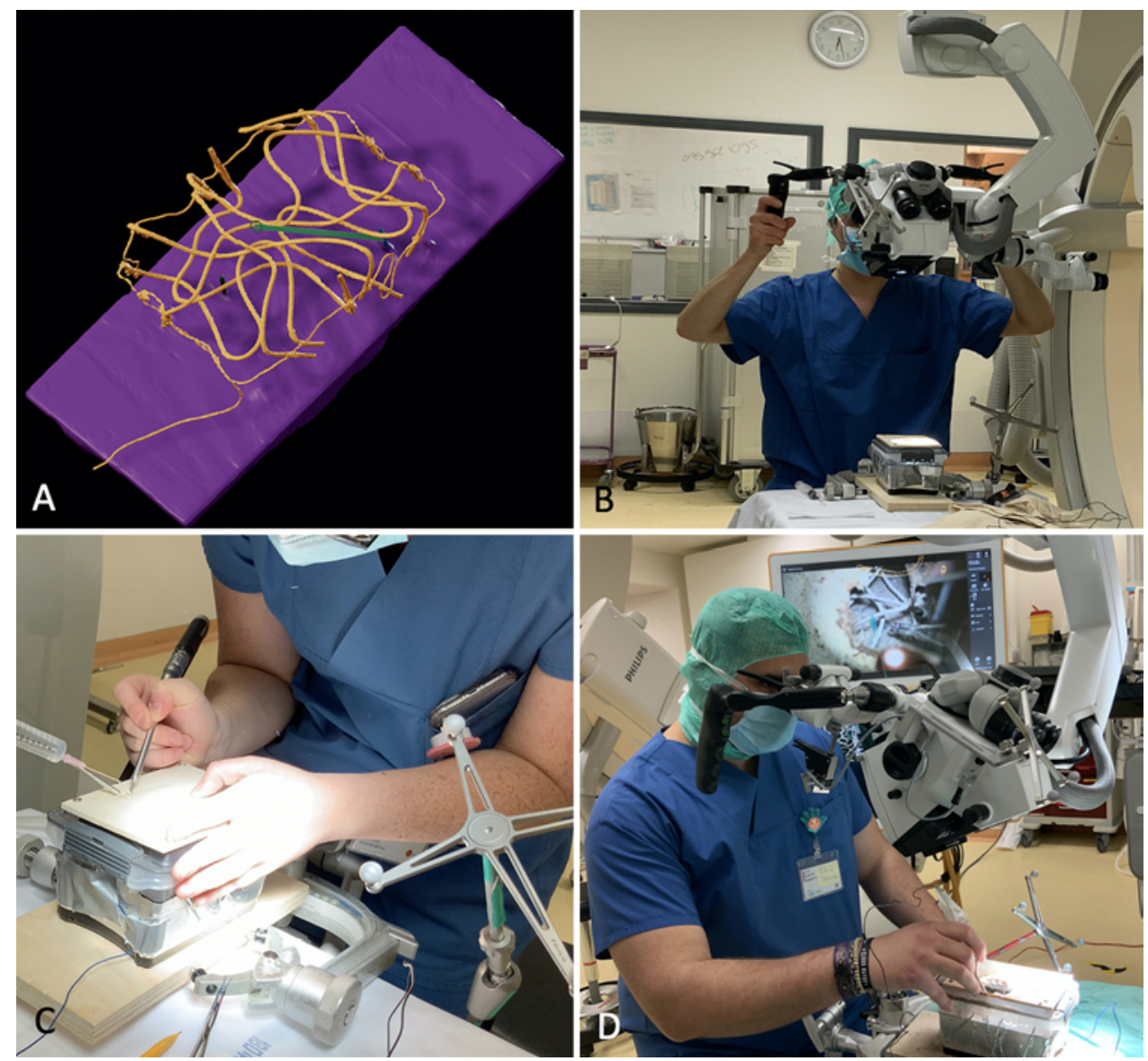

FIG. 3. The safety task using AR. A: Participants were first asked to define, on the navigation system, an ideal trajectory between the wooden top of the box and the target, avoiding the metal wires (green line). B: The surgical microscope should be aligned with the formed trajectory. C: The wooden top of the box was drilled according to AR-assisted navigation. D: Under the microscope, participants were asked to clip the target (nail) without touching the metal wires with the help of AR.

\section{Outcome}

The primary outcome was the number of contacts between the clip applier and the metallic wires inside the connected box. Secondary outcomes were the size of the craniotomy expressed in square centimeters, successful clipping of the hidden nail, the number of attempts, and the subjective trust in the navigation system.

\section{Results}

\section{Participants}

Of the 23 participants, 6 (26.1\%) were female. None of the participants had experience with NV or AR, and none had experience with an aneurysm clipping procedure. All participants could perform the safety tasks first with the use of NV and then with the use of AR.

\section{Number of Contacts and Size of the Craniotomy}

All measures regarding the number of contacts between the aneurysm clip applier and the metal wires were successfully recorded. The number of contacts tended to be lower when using AR compared with NV (AR, median 1 [Q1: 1, Q3: 2]; NV, median 3 [Q1: 1, Q3: 6]; p = 0.074)
(Fig. 4). When counting only participants who successfully clipped the nail, no difference was found between AR and NV (AR, median 1 [Q1: 1, Q3: 2]; NV, median 1 [Q1: 1, Q3: 4.5]; $\mathrm{p}=0.45$ ). The surface of the craniotomy was significantly smaller when using AR compared with NV (AR, median $4.91 \mathrm{~cm}^{2}$ [Q1: $4.71 \mathrm{~cm}^{2}$, Q3: $7.55 \mathrm{~cm}^{2}$ ]; NV, median $9.62 \mathrm{~cm}^{2}$ [Q1: $7.07 \mathrm{~cm}^{2}$, Q3: $13.85 \mathrm{~cm}^{2}$ ]) (Fig. 5).

\section{Success Rate and Number of Attempts}

Nineteen participants (82.6\%) successfully clipped the nail using AR compared with 15 participants (65.2\%) using NV. A statistical significance was not reached with regard to the clipping success rate $(p=0.31)$. The number of attempts did not differ significantly between AR and NV (AR, median 2 [Q1: 1, Q2: 2]; NV, median 2 [Q1: 1.5, Q3: 3.5]; $\mathrm{p}=0.17$ ).

\section{Subjective Trust in the Navigation Systems}

No difference was found in the pretest trust between AR and NV (AR, mean 7.3 [SD 1.3]; NV, mean 7.1 [SD 1.1]; $\mathrm{p}=0.63)$. The posttest trust for AR was higher than for NV (AR, mean 8.2 [SD 0.9]; NV, mean 6.8 [SD 2.5]; 


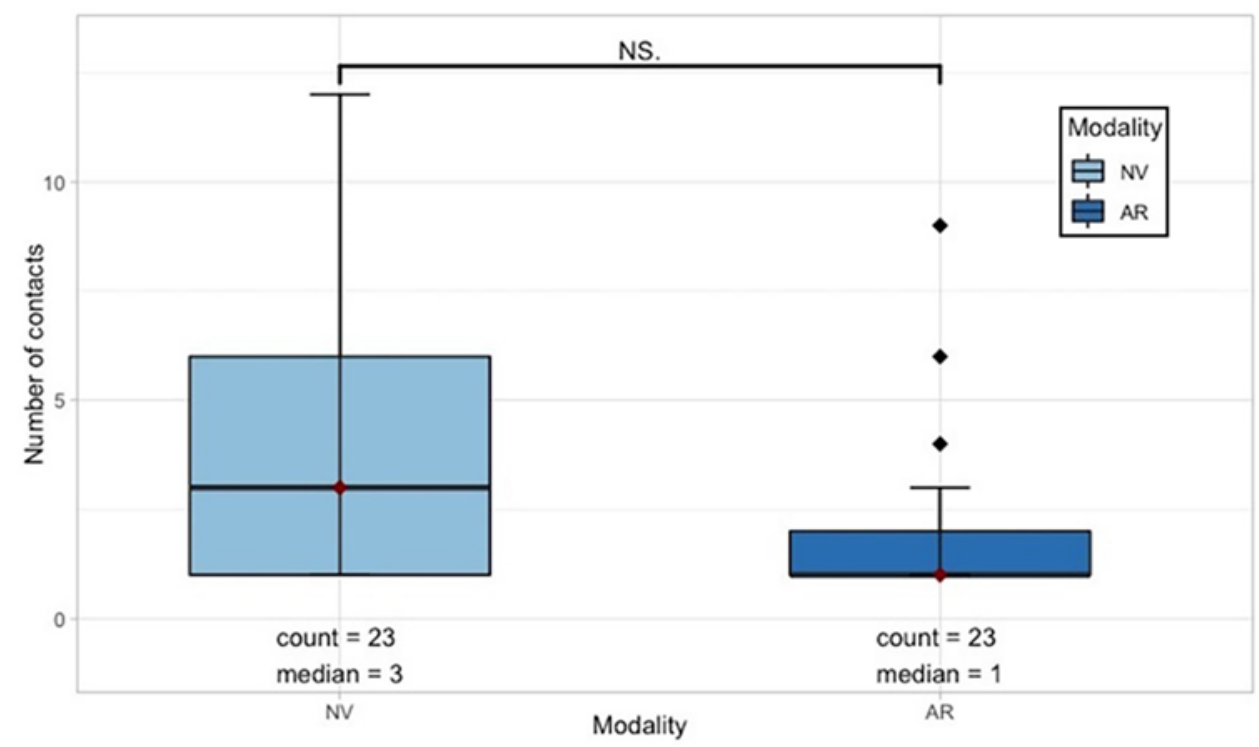

FIG. 4. Boxplot showing the median number of contacts between the connected clip applier and the metal wires inside the box ( $p=$ 0.074). NS. = not significant.

$p=0.013)$. The difference in posttest trust minus pretest trust between AR and NV was statistically significant in favor of AR (AR, mean 0.9 [SD 1.2]; NV, mean -0.3 [SD 0.2 ]; $\mathrm{p}<0.05$ ) (Fig. 6).

\section{Discussion}

In a model mimicking a typical restricted microsurgical corridor (e.g., a sylvian fissure), the number of contacts between the clip applier and perifocal metal wires tended to be less with the use of AR than with NV. Furthermore, the use of AR gave a significantly smaller craniotomy.
Although the number of attempts and the success rate to clip the target did not differ between the two navigation techniques, participants expressed more trust in AR after using it in this setup.

Preoperative evaluation of the surgical strategy is mandatory to achieve the surgical goal and to avoid collateral damages. For example, a vein-preserving sylvian fissure dissection is important to avoid venous infarction, which can dramatically affect patient outcomes. ${ }^{16,17}$ Neuronavigation systems can be used to help anticipate surgical trajectories. Our model is, to the best of our knowledge, the first attempt to assess vascular integrity using a sound

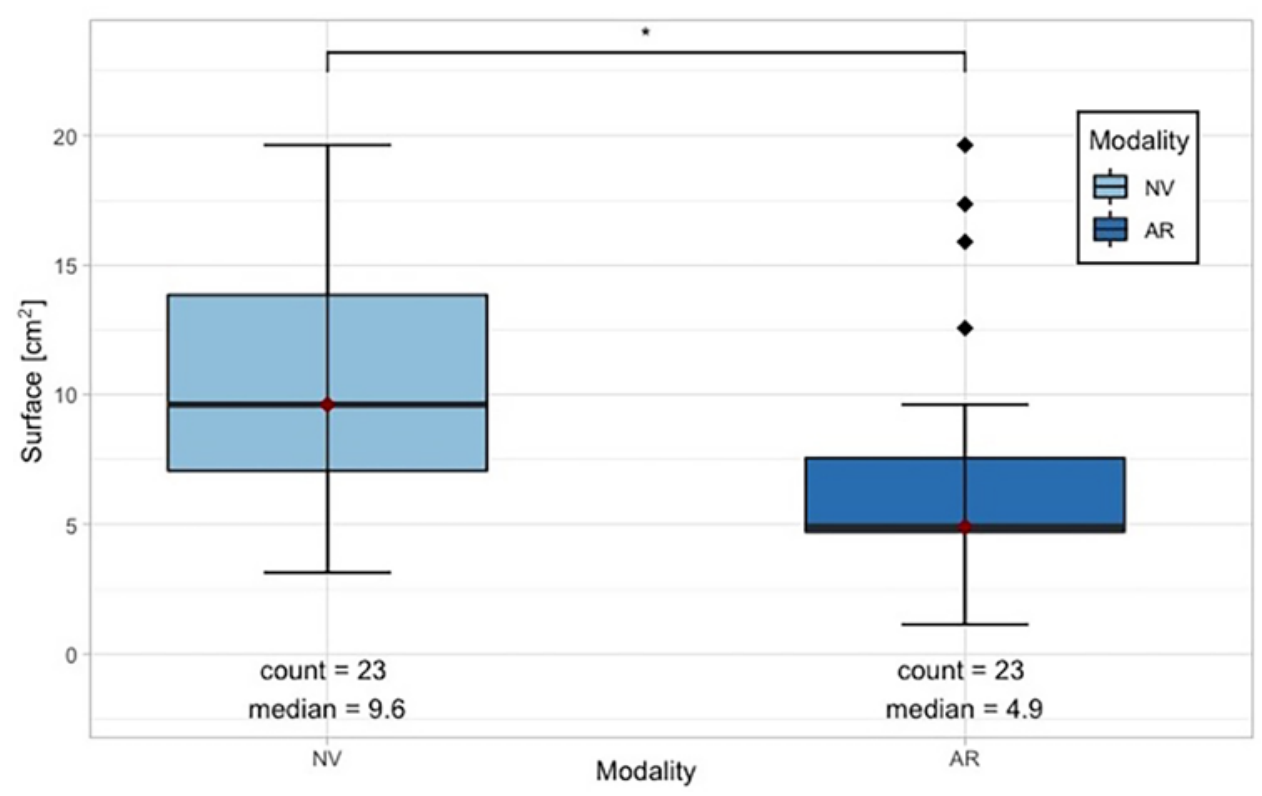

FIG. 5. Boxplot presenting the median size of the craniotomy (* $p<0.05)$. 


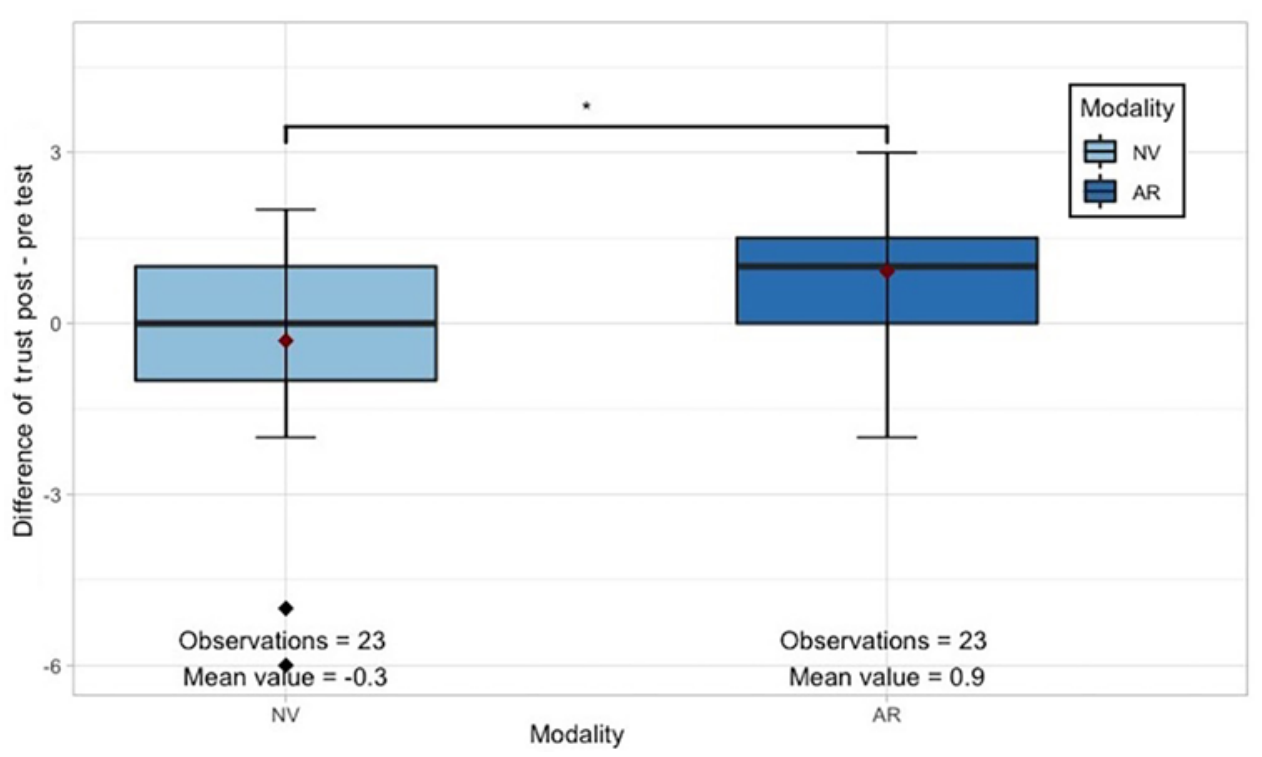

FIG. 6. Boxplot showing the difference of trust posttest minus pretest $\left({ }^{*} p<0.05\right)$.

alert if there is any contact between the surgical instrument and a metal wire representing an intracranial vessel. In this experiment, the use of AR tended to reduce the number of contacts compared with the NV-guided approach. Defining an ideal trajectory preoperatively helped the participants not only to choose the safest way to reach the target, but also to have a better overview of the courses of the vessels. This is emphasized by preoperative trajectory-guided deep brain electrode insertions or biopsy using frameless neuronavigation systems..$^{18,19}$ The difference between AR and NV did not, however, reach significance. This may be explained by the fact that we only tested naive participants to avoid a bias linked to previous microsurgical experience, or due to a lack of study power. The same scenario should be tested in experienced neurosurgeons to assess whether such changes remain present in experienced hands. Second, the connected box was not filled, so no dissection was needed. Participants had a direct view of the wires through the surgical microscope and used less of the perspective effect that AR offers.

The size of the craniotomy positively affects patient outcome, ${ }^{20-22}$ and, in our study, the median size of the craniotomy was significantly smaller with the use of AR. Di Bonaventura et al. ${ }^{21}$ analyzed intraoperative and postoperative complications after a minipterional craniotomy compared with standard pterional craniotomy. ${ }^{21}$ The authors reported less intraoperative complication, less postoperative seizure, and a shorter hospitalization in the minipterional group than in the standard pterional craniotomy group. In the current study, AR offered the advantage of being able to align the microscope along the chosen trajectory. Thereby, participants could drill around the entry point and used AR to evaluate the necessary space required to insert the clip applier to clip the nail. With the use of NV, participants estimated an a priori reasonable craniotomy surface without direct visual feedback.

As discussed in a previous publication, ${ }^{2}$ we chose to test participants (students) naive to navigation systems and sur- gery to avoid bias linked to previous experience. All participants succeeded in using the two navigation systems as well as the AR-guided surgical microscope after a short introduction, indicating that AR and NV are easy to use. The first AR task of defining a trajectory was also an important step of the process as participants encountered the first 3D representation of the metal wires inside the box, a step that anticipated a future real surgical experience. The novel development of mixed reality, or the combination of virtual and physical components, opens new perspectives in surgical planning for cranial and spine surgery. ${ }^{23,24}$

\section{Limitations}

The main limitation of this study is that all participants started the safety tasks using NV and then repeated the same task using AR, something that obviously led to learning. Consequently, the part of the learning phase concerning the reduction of the number of contacts remains unknown. Randomization of participants according to neuronavigation techniques will be conducted in the future. Second, our model was a plastic box customized for the purpose. It does not contain the typical anatomical structures and properties normally encountered during a traditional sylvian exposure, and an actual surgical environment involves dynamic changes, which were not possible here. Finally, despite mimicking surgical conditions as accurately as possible, it remains a laboratory experiment, and assessment of the integrity of the perifocal structures and the trust in AR versus NV need to be formally assessed during a cranial approach in future studies.

\section{Conclusions}

The comparison of the use of NV and AR in a model mimicking a typical restricted surgical corridor with vessels passing through showed a trend favoring the use of AR-guided navigation with respect to the surrounding structures. AR offers the possibility to define a surgical 
strategy and an ideal trajectory by using real-time projections of virtual objects on real structures through the surgical microscope.

\section{References}

1. Bir SC, Konar SK, Maiti TK, Thakur JD, Guthikonda B, Nanda A. Utility of neuronavigation in intracranial meningioma resection: a single-center retrospective study. World Neurosurg. 2016;90:546-555.e1.

2. Haemmerli J, Davidovic A, Meling TR, Chavaz L, Schaller $\mathrm{K}$, Bijlenga P. Evaluation of the operative augmented reality precision compared to standard neuronavigation using a 3Dprinted skull. Neurosurg Focus. 2021;50(1):E17.

3. Jenkinson MD, Barone DG, Bryant A, Vale L, Bulbeck H, Lawrie TA, et al. Intraoperative imaging technology to maximise extent of resection for glioma. Cochrane Database Syst Rev. 2018;1:CD012788.

4. Legninda Sop FY, D'Ercole M, Izzo A, Rapisarda A, Ioannoni E, Caricato A, et al. The impact of neuronavigation on the surgical outcome of microvascular decompression for trigeminal neuralgia. World Neurosurg. 2021;149:80-85.

5. Spetzger U, Laborde G, Gilsbach JM. Frameless neuronavigation in modern neurosurgery. Minim Invasive Neurosurg. 1995;38(4):163-166.

6. Meola A, Cutolo F, Carbone M, Cagnazzo F, Ferrari M, Ferrari V. Augmented reality in neurosurgery: a systematic review. Neurosurg Rev. 2017;40(4):537-548.

7. Lavé A, Meling TR, Schaller K, Corniola MV. Augmented reality in intracranial meningioma surgery: report of a case and systematic review. J Neurosurg Sci. 2020;64(4):369-376.

8. Lee C, Wong GKC. Virtual reality and augmented reality in the management of intracranial tumors: a review. J Clin Neurosci. 2019;62:14-20.

9. Cabrilo I, Bijlenga P, Schaller K. Augmented reality in the surgery of cerebral aneurysms: a technical report. Neurosurgery. 2014;10(suppl 2):252-261.

10. Cabrilo I, Bijlenga P, Schaller K. Augmented reality in the surgery of cerebral arteriovenous malformations: technique assessment and considerations. Acta Neurochir (Wien). 2014; 156(9):1769-1774.

11. Cabrilo I, Schaller K, Bijlenga P. Augmented reality-assisted bypass surgery: embracing minimal invasiveness. World Neurosurg. 2015;83(4):596-602.

12. Rychen J, Goldberg J, Raabe A, Bervini D. Augmented reality in superficial temporal artery to middle cerebral artery bypass surgery: technical note. Oper Neurosurg (Hagerstown). 2020;18(4):444-450.

13. Carl B, Bopp M, Saß B, Nimsky C. Microscope-based augmented reality in degenerative spine surgery: initial experience. World Neurosurg. 2019;128:e541-e551.

14. Carl B, Bopp M, Saß B, Pojskic M, Nimsky C. Augmented reality in intradural spinal tumor surgery. Acta Neurochir (Wien). 2019;161(10):2181-2193.

15. Molina CA, Dibble CF, Lo SL, Witham T, Sciubba DM. Augmented reality-mediated stereotactic navigation for execution of en bloc lumbar spondylectomy osteotomies. J Neurosurg Spine. 2021;34(5):700-705.

16. Maekawa H, Hadeishi H. Venous-preserving sylvian dissection. World Neurosurg. 2015;84(6):2043-2052.
17. Suzuki Y, Endo T, Ikeda H, Ikeda Y, Matsumoto K. Venous infarction resulting from sacrifice of a bridging vein during clipping of a cerebral aneurysm: preoperative evaluation using three-dimensional computed tomography angiographycase report. Neurol Med Chir (Tokyo). 2003;43(11):550-554.

18. Ladisich B, Machegger L, Romagna A, Krainz H, Steinbacher J, Leitinger M, et al. VarioGuide frameless neuronavigation-guided stereoelectroencephalography in adult epilepsy patients: technique, accuracy and clinical experience. Acta Neurochir(Wien). 2021;163(5):1355-1364.

19. Satoh M, Nakajima T, Yamaguchi T, Watanabe E, Kawai K. Application of augmented reality to stereotactic biopsy. $\mathrm{Neu}$ rol Med Chir (Tokyo). 2019;59(11):444-447.

20. Caplan JM, Papadimitriou K, Yang W, Colby GP, Coon AL, Olivi A, et al. The minipterional craniotomy for anterior circulation aneurysms: initial experience with 72 patients. Neurosurgery. 2014;10(suppl 2):200-207.

21. Di Bonaventura R, Sturiale CL, Latour K, Mazzucchi E, Marchese E, Albanese A. Comparison between minipterional craniotomy associated with focused sylvian fissure opening and standard pterional approach with extended sylvian fissure dissection for treatment of unruptured middle cerebral artery aneurysms. World Neurosurg. 2021;146:e1293-e1300.

22. Thakur JD, Mallari RJ, Corlin A, Yawitz S, Huang W, Eisenberg A, et al. Minimally invasive surgical treatment of intracranial meningiomas in elderly patients ( $\geq 65$ years): outcomes, readmissions, and tumor control. Neurosurg Focus. 2020;49(4):E17.

23. Neves CA, Vaisbuch Y, Leuze C, McNab JA, Daniel B, Blevins NH, Hwang PH. Application of holographic augmented reality for external approaches to the frontal sinus. Int Forum Allergy Rhinol. 2020;10(7):920-925.

24. Yuk FJ, Maragkos GA, Sato K, Steinberger J. Current innovation in virtual and augmented reality in spine surgery. Ann Transl Med. 2021;9(1):94.

\section{Disclosures}

The authors report no conflict of interest concerning the materials or methods used in this study or the findings specified in this paper.

\section{Author Contributions}

Conception and design: Haemmerli, Bijlenga. Acquisition of data: Haemmerli, Davidovic, Chavaz. Analysis and interpretation of data: Haemmerli, Bijlenga. Drafting the article: Haemmerli, Davidovic, Chavaz. Critically revising the article: Meling, Schaller, Bijlenga. Reviewed submitted version of manuscript: Haemmerli, Meling, Bijlenga. Approved the final version of the manuscript on behalf of all authors: Haemmerli. Statistical analysis: Haemmerli. Administrative/technical/material support: Haemmerli, Davidovic, Chavaz. Study supervision: Haemmerli, Bijlenga.

\section{Correspondence}

Julien Haemmerli: Geneva University Hospitals, Geneva, Switzerland. julien.haemmerli@hcuge.ch. 